\section{Impacto da Estratégia Saúde da Família sobre indicadores de saúde bucal: análise em municípios do Nordeste brasileiro com mais de 100 mil habitantes}

\author{
Impact of the Family Health Strategy: an analysis \\ in cities in Northeast Brazil with more than \\ 100,000 inhabitants
}

\author{
1 Pontifícia Universidade \\ Católica de Minas Gerais, \\ Belo Horizonte, Brasil. \\ 2 Departamento de \\ Odontologia, Universidade \\ Federal do Rio Grande do \\ Norte, Natal, Brasil. \\ ${ }^{3}$ Faculdade de Odontologia, \\ Universidade Federal da \\ Bahia, Salvador, Brasil. \\ ${ }_{4}$ Conselho Regional de \\ Odontologia do Maranhão, \\ São Luís, Brasil. \\ Correspondência \\ C. R. S. Pereira \\ Pontifícia Universidade \\ Católica de Minas Gerais. \\ Rua Prof. Raphael Hardy 126 , \\ Belo Horizonte, $M G$ \\ 30730-080, Brasil. \\ carmenpuc@ig.com.br
}

\begin{abstract}
This study aimed to analyze the impact of the Family Health Strategy (FHS) on oral health in Northeast Brazil, in 12 municipalities with more than 100 thousand inhabitants. The reference was 20 census tracts, 10 of which in areas with oral health covered by the FHS for at least a year, matched with 10 census tracts without oral health coverage. The total sample included 59,221 individuals. Three possible effects were considered: negative, positive, and absence of effect on the outcomes, based on the prevalence ratio adjusted by Poisson regression. The outcomes "access to collective preventive measures" and "treatment of toothache" indicate a positive effect in the FHS oral health model. However, the outcomes "coverage of tooth extraction" and "restorative treatment" showed either a negative effect or no effect in the majority of the municipalities in the sample. Evidence indicates that within the $F H S$, the results reflect the local organizational models for oral health care.
\end{abstract}

Family Health Programs; Oral Health; Health Services Evaluation
Carmen Regina dos Santos Pereira 1

Angelo Giuseppe Roncalli 2

Maria Cristina Teixeira Cangussu 3

Luiz Roberto Augusto Noro 2

Alberto Allan Rodrigues Patrício 4

Kenio Costa Lima ${ }^{2}$

\section{Introdução}

O desenvolvimento técnico-científico e as transformações político-econômicas que caracterizam as sociedades capitalistas ocidentais reforçam a necessidade de implementação de sistemas de atenção à saúde cada vez mais amplos e complexos. Nessa perspectiva, o acúmulo de conhecimento existente em Saúde Coletiva, obtido, entre outros aspectos, por meio das experiências bem sucedidas no país, tem ajudado no desenvolvimento de métodos e tecnologias que deem suporte ao pleno desenvolvimento do Sistema Único de Saúde (SUS). Para tanto, a investigação em serviços de saúde assume especial relevância, pois um dos seus objetivos é orientar o desenho de políticas e a melhoria do seu desempenho ${ }^{1}$.

O SUS, por suas características doutrinárias e organizacionais, tem norteado as políticas de assistência à saúde no Brasil ${ }^{2}$. Nesse contexto, a Estratégia Saúde da Família (ESF) busca incorporar efetivamente os princípios de universalidade, integralidade e equidade. No tocante à Política de Saúde Bucal, as ações passaram a integrar as atividades do ESF por meio da Portaria GM/MS $n^{o}$. 1.444 de $2000^{3}$, a qual estabelece o incentivo para a implantação das equipes de saúde bucal (ESB) no Programa Saúde da Família (PSF). A presença da ESB no PSF visa ampliar o acesso coletivo às ações de promoção, prevenção e recuperação da saúde bucal e a consequente melhoria de seus indicadores epidemiológicos. 
Foram implantadas na ESF, de dezembro de 2002 a abril de 2010, 19.488 equipes de saúde bucal que atuam em 4.753 municípios, atingindo $71 \%$ de cobertura de toda a população brasileira (DATASUS. Informações em saúde. http://www. datasus.gov.br/DATASUS/DATASUS.php, acessado em 30/Jun/2010). Tamanho investimento requer, por sua vez, esforços no sentido de monitorar e avaliar seus impactos, os quais constituem a abordagem deste trabalho.

Tendo como objeto a ESF, diversos estudos avaliando seu impacto sobre indicadores de saúde foram realizados desde sua implantação em meados dos anos 90. Uma maior produção tem sido dedicada a indicadores passíveis de sofrerem efeitos por estratégias de atenção primária, como a mortalidade infantil. Utilizando dados de séries temporais entre 1999 e 2004, no Brasil, Macinko et al. 4 avaliaram as taxas de mortalidade infantil (TMI) em 557 microrregiões, buscando a associação com a cobertura de PSF e controlando por variáveis socioeconômicas e de serviços. Encontraram uma relação significativa entre o crescimento do PSF e o declínio na mortalidade infantil. Resultados semelhantes foram encontrados por Aquino et al. ${ }^{5}$. Nesse estudo, após o controle dos fatores confundidores, foram observadas reduções de $13 \%$ a $22 \%$ na TMI, para níveis igualmente crescentes de cobertura pelo PSF.

Ainda no campo da saúde da criança, Monteiro et al. 6 observaram um declínio considerável na desnutrição infantil entre 1996 e 2006 e observaram que parte do efeito poderia ser creditado à assistência à saúde. Para os autores, “a expansão do acesso de mães e crianças à assistência à saúde coincide com a expansão no País do Programa de Saúde da Família (PSF), cuja proposta enfatiza a prevenção e a educação em saúde" 6 (p. 42).

Em um amplo estudo realizado em 2009, Rocha \& Soares 7 avaliaram o impacto do PSF em uma série de indicadores, associando-os com dados de cobertura do programa em municípios brasileiros de 1993 a 2004. Encontraram resultados positivos para mortalidade infantil e, ainda, sobre o trabalho infantil e a escolaridade em crianças.

Um outro aspecto frequentemente avaliado é o efeito sobre internações hospitalares por condições sensíveis à Atenção Primária em Saúde. Nesse sentido, Veloso \& Araújo 8 observaram um declínio de 43\% em 1999 para 29\% em 2007 nas internações sensíveis em municípios de Minas Gerais. Apesar de o estudo não ter controlado o efeito de outros fatores, as autoras creditam parte desse efeito à coincidente expansão do PSF no mesmo período.

Boa parte dos estudos que avalia o efeito do PSF toma, como unidade de análise, os municí- pios participantes ou não do programa ou, ainda, com diferentes taxas de cobertura. Tais estudos carregam um viés intrínseco aos estudos de delineamento ecológico, particularmente quando se buscam associações entre fatores condicionantes e indicadores epidemiológicos ou de serviços. Outra abordagem, que controla mais adequadamente esse viés, tem sido utilizada em estudos que levam, em conta, a comparação, em um mesmo município, de áreas cobertas ou não cobertas pelo programa. Goldbaum et al. ${ }^{9}$ optaram por esse modelo de análise quando avaliaram a utilização de serviços de saúde em dois distritos de saúde no Município de São Paulo. Os autores encontraram que "a presença do PSF mostrou-se capaz de diminuir o efeito das condições sociais desiguais sobre o perfil de acesso e utilização ali existente" 9 (p. 98).

No campo da saúde bucal, os estudos avaliativos da ESF ainda são menos frequentes, e poucos deles abordam diretamente o impacto sobre indicadores epidemiológicos ou de serviços. Baldani et al. 10 analisaram o perfil de implantação da Saúde Bucal no PSF em 136 municípios do Estado do Paraná, um ano após a vigência da Portaria $n^{\circ} .1 .444$, e encontraram pontos positivos no sentido da recepção favorável por parte da população e participação dos cirurgiões-dentistas em cursos de capacitação e já antecipava a questão do nó crítico do encaminhamento dos casos complexos. Andrade \& Ferreira 11, Oliveira \& Saliba 12 e Santos \& Assis 13 também identificaram que ações individuais e coletivas da ESB apresentaram resolutividade limitada nos municípios de Campos de Goytacazes (Rio de Janeiro), Recife (Pernambuco) e Alagoinhas (Bahia), respectivamente.

Na análise do modelo de atenção à saúde bucal em nove municípios de Pernambuco, sob o ponto de vista dos coordenadores municipais, associada ao estudo do perfil da ESB, constatouse que a maioria dos municípios apresentou dificuldades na estruturação do modelo de prática, tanto do ponto de vista da incorporação de novas práticas, como na deficitária qualificação dos profissionais 14 .

Souza \& Roncalli 15 , numa tentativa de identificar, em 19 municípios do Rio Grande do Norte, os fatores capazes de interferir no processo de mudança dos modelos assistenciais em saúde, concluíram que, devido à precariedade nas relações de trabalho e dificuldades no referenciamento para os níveis de maior complexidade, a maioria dos municípios apresentou pouco ou nenhum avanço no modelo assistencial.

Assim, pode-se considerar que a produção de evidências sobre o efeito das ações de saúde bucal na ESF ainda é muito escassa. Nesse sen- 
tido, o objetivo do presente estudo foi verificar se a incorporação da equipe de saúde bucal na ESF gerou impacto sobre indicadores de saúde bucal relativos à prevalência de agravos e sua respectiva cobertura de tratamento, bem como ao acesso ao serviço público e às ações preventivas em saúde bucal. Tomou-se, como referência, a população nordestina residente em municípios com mais de 100 mil habitantes.

\section{Método}

\section{Tipo de estudo}

Trata-se de um estudo observacional, de base populacional e de arquitetura transversal 17 .

\section{Plano amostral}

\section{- Definição dos domínios de estudo}

Foram identificados, dentre os municípios da Região Nordeste com mais de 100 mil habitantes, aqueles que apresentavam equipes de saúde bucal na ESF. Segundo dados do Sistema de Informações da Atenção Básica (SIAB), disponibilizados pela Coordenação Nacional de Saúde Bucal, para setembro de 2005, 48 municípios do Nordeste com mais de 100 mil habitantes possuíam equipes de saúde bucal na ESF com distintos graus de cobertura, tanto para a modalidade I, quanto para a modalidade II de incorporação e com tempo de cobertura superior a um ano (DATASUS. Pacto da Atenção Básica. http://tab net.datasus.gov.br/cgi/siab/pacto2005/pcamap. htm, acessado em 29/Set/2005).

Considerando que uma cobertura total, bem como uma baixa cobertura não seriam adequadas para um estudo comparativo pela clara impossibilidade de obtenção de áreas-controle, optou-se por um corte arbitrário de modo a incluir somente municípios com, no mínimo, $20 \%$ e, no máximo, $70 \%$ de cobertura de saúde bucal da ESF. Vinte e seis, dos 48 municípios, preencheram esses critérios e foram convidados a participar do estudo. Ao final, 12 municípios que compõem a amostra concluíram todas as etapas. Os demais municípios deixaram de participar por vontade do gestor, pela demora no retorno dos dados solicitados ou por problemas de ordem operacional.

\section{- Definição das Unidades Primárias de Amostragem (UPA)}

Foi utilizado, como Unidade Primária de Amostragem (UPA), o setor censitário, uma vez que se trata de uma unidade de análise que contém informações socioeconômicas que permitem emparelhar as áreas que estão sendo comparadas. Considerando que os limites dos setores censitários não seguem a mesma lógica de delimitação das áreas de cobertura da ESF com saúde bucal, tais áreas foram sobrepostas aos mapas de setores censitários de modo a identificar aqueles total ou parcialmente cobertos e os totalmente descobertos pela ESF. Os setores parcialmente cobertos foram excluídos, os totalmente cobertos foram considerados como grupo experimental, e os não cobertos, como grupo controle.

Uma vez estabelecido o universo a ser incluído, foram sorteados dez setores das áreas cobertas pela ESB na ESF há, pelo menos, um ano. Para compor as áreas sem cobertura (controles), foi realizado um emparelhamento intencional a partir dos dados socioeconômicos constantes nas bases de dados dos setores censitários. Foram selecionadas nove variáveis socioeconômicas, as quais compuseram um índice classificatório final para que o emparelhamento pudesse ser realizado. Essas variáveis pertenciam a três dimensões: (a) educacional, formada pelas variáveis "escolaridade do chefe de família", "percentual de adultos analfabetos" e "percentual de crianças de 5 anos analfabetas"; (b) econômica, composta pelas variáveis "renda média do chefe do domicílio", "percentual de responsáveis desempregados" e "percentual de responsáveis com renda inferior a um salário mínimo"; e (c) sanitária, composta pelas variáveis "acesso a água tratada", "percentual de domicílios sem banheiro" e "percentual de domicílios com lixo coletado” 17.

\section{- Tamanho da amostra}

Tomou-se, como base, o efeito presumido da saúde bucal na ESF sobre um dos desfechos utilizados no estudo, o acesso a serviço odontológico público, medido pela consulta ao dentista nos 12 meses anteriores à pesquisa. Os dados do estudo feito em Natal (Rio Grande do Norte) foram utilizados como referência. De acordo com os resultados, a consulta ao dentista em áreas com modelo tradicional ou sem cobertura foi de $27,1 \%$. Admitiu-se um efeito presumido da saúde bucal no PSF de $20 \%$, o qual, associado a um nível de significância $(\alpha)$ de 0,05 e um poder ( $\beta$ ) de $90 \%$, de acordo com a técnica preconizada por Altman 18 e também utilizada por Roncalli \& Lima 19, gera um tamanho inicial de amostra de 300 indivíduos. Por se tratar de um delineamento com múltiplos estágios, foi estabelecido um efeito de desenho de 1,5, e, ainda, estimada uma taxa de não-resposta de $20 \%$, o que resultou em uma amostra final de 495 indivíduos. 
Tendo em conta, ainda, o efeito da idade sobre o desfecho "acesso ao serviço odontológico", uma pré-estratificação foi realizada, considerando os grupos etários de 0 a 12 anos (crianças), 13 a 19 (adolescentes), 20 a 59 (adultos) e 60 anos ou mais (idosos). Tomou-se, como base, a proporção da população em cada uma dessas faixas etárias, em cada um dos 12 municípios da amostra, considerando, ainda, que um mínimo de 495 indivíduos fosse pesquisado na faixa etária de 13 a 19 anos. Desse modo, o tamanho final da amostra, em cada grupo, ficou em torno de 3 mil indivíduos, totalizando cerca de 6 mil indivíduos a serem examinados em cada município. Considerando os 12 municípios estudados, a amostra final prevista foi de 37.574 em cada grupo (coberto ou não coberto), totalizando 75.148 indivíduos.

\section{- Processo de alocação dos elementos amostrais}

O número de domicílios percorridos para que se pudesse obter o número final de amostra foi estabelecido a partir da média de moradores do domicílio. O segundo estágio, o qual consiste no sorteio dos domicílios a serem pesquisados, seguiu a metodologia semelhante às pesquisas para avaliação de cobertura vacinal, proposta pela Organização Mundial da Saúde (OMS) e descrita por Barros \& Victora 20.

\section{Estratégias e instrumentos de coleta de dados}

Todos os indivíduos participantes do estudo foram entrevistados em suas residências por Agentes Comunitários de Saúde (ACS) devidamente capacitados em oficinas de trabalho com 12 horas de duração. Essas oficinas abordaram as técnicas de reconhecimento da área e dos domicílios, assim como o preenchimento do questionário-entrevista desenvolvido para a pesquisa. Esse questionário-entrevista foi aplicado pelo ACS junto ao informante mais qualificado do domicílio, sendo, portanto, um instrumento que agregou informações relativas a todos os moradores.

\section{Variáveis}

A variável de interesse explanatória é a presença de saúde bucal na ESF da área. As variáveis de resposta (dependentes) são as relativas à situação de saúde bucal e que possuem a capacidade de serem influenciadas pela presença da ESB: morbidade dentária autorreferida, prevalência de dor de origem dentária, tratamento da dor de origem dentária, consulta ao serviço odontológico no último ano, tratamento restaurador ou mutilador e ações preventivas individuais ou coletivas (Tabela 1).

Pelo fato de as áreas pesquisadas terem sido emparelhadas, as variáveis "número de bens", "renda per capita" e "escolaridade da mãe" passam a se comportar como variáveis confundidoras e não explicativas do efeito da variável principal.

\section{Análise dos dados}

Para a análise do efeito sobre os desfechos, foram calculadas medidas de associação, relacionando as frequências entre as áreas estudadas. A magnitude dos efeitos foi verificada pelo cálculo da razão de prevalência (RP) e seus respectivos intervalos de 95\% de confiança (IC95\%), ajustada para os fatores de confusão, por intermédio da regressão de Poisson com variância robusta. O limite de significância foi estabelecido para um $\alpha=0,05$.

\section{Estratégia de análise}

Nos 12 municípios, todas as ESB eram constituídas de Cirurgião-dentista (CD) e Auxiliar de Saúde Bucal (ASB) (Modalidade 1). A inserção dessas ESB ocorreu, de modo geral, de forma gradual, gerando situações de três tipos: (a) ESB trabalhando integradas às ESF, (b) CD em unidades com Programa de Agentes Comunitários de Saúde (PACS) ou ESF, mas não integrados a esse novo processo de trabalho, (c) CD nas Unidades Básicas de Saúde (UBS) sem a presença do PACS ou ESF. Portanto, por essa particularidade, na análise dos dados com relação à variável independente principal, o tipo de cobertura foi estabelecido do seguinte modo:

Modelo 1 (Grupo Experimental): caracterizase pela efetiva implantação da ESB na ESF com tempo mínimo de 1 ano;

Modelo 2 (Grupo Controle): caracteriza-se pela existência de CD lotado em unidades com ESF ou PACS, mas sem vinculação a um ou outro modelo assistencial, trabalhando numa organização de trabalho "não-ESF" (modelo tradicional) ou ainda em áreas sem nenhuma assistência.

\section{Aspectos éticos}

O projeto de pesquisa foi submetido ao Comitê de Ética em Pesquisa da Universidade Federal do Rio Grande do Norte para a devida análise, recebendo parecer favorável à sua execução, estando registrado no Sistema Nacional de Ética em Pesquisa (SISNEP) sob número 0028.1.051.000- 
Elenco de variáveis dependentes e independentes do estudo.

\begin{tabular}{|c|c|}
\hline Variáveis & Descrição \\
\hline \multicolumn{2}{|l|}{ Independentes } \\
\hline \multicolumn{2}{|l|}{ Principal } \\
\hline Presença de ESB na ESF & Presença ou ausência de cobertura pela ESB na ESF \\
\hline \multicolumn{2}{|l|}{ Confundidoras } \\
\hline Número de bens & $\begin{array}{l}\text { Número de bens por domicílio dicotomizados a partir da mediana, formando } \\
\text { as categorias: "desfavorável" }(<4) \text { e "favorável" }(\geq 4)\end{array}$ \\
\hline Renda per capita & $\begin{array}{l}\text { Renda em reais do chefe da família no último mês dicotomizada a partir da } \\
\text { mediana, formando as categorias: "desfavorável" }(<\mathrm{R} \$ 112,50) \text { e "favorável" } \\
(\geq \mathrm{R} \$ 112,50)\end{array}$ \\
\hline Escolaridade da mãe & $\begin{array}{l}\text { Anos de estudo da mãe dicotomizados a partir da mediana, formando as } \\
\text { categorias: "desfavorável" (<4 anos) e "favorável" ( } \geq 4 \text { anos) }\end{array}$ \\
\hline \multicolumn{2}{|l|}{ Dependentes } \\
\hline \multicolumn{2}{|l|}{ Indicadores de saúde bucal } \\
\hline Acesso a serviço odontológico & $\begin{array}{l}\text { Frequência de consulta ao dentista no último ano obtida através da pergunta } \\
\text { "Foi ao dentista da sua unidade de saúde nos últimos } 12 \text { meses?" }\end{array}$ \\
\hline $\begin{array}{l}\text { Prevalência de dor de origem } \\
\text { dentária }\end{array}$ & $\begin{array}{l}\text { Prevalência de dor de origem dentária obtida através da pergunta } \\
\text { "Teve dor de dente nos últimos } 12 \text { meses?" }\end{array}$ \\
\hline Morbidade dentária autorreferida & $\begin{array}{l}\text { Prevalência obtida através da pergunta: "Acha que necessita de tratamento } \\
\text { dentário atualmente?" }\end{array}$ \\
\hline $\begin{array}{l}\text { Tratamento de dor de origem } \\
\text { dentária }\end{array}$ & $\begin{array}{l}\text { Frequência obtida através da pergunta feita aos que tiveram dor de dente: } \\
\text { "Foi tratado no serviço público local?" }\end{array}$ \\
\hline Tratamento restaurador & $\begin{array}{l}\text { Prevalência obtida através da pergunta "Fez obturação na sua unidade } \\
\text { de saúde?" }\end{array}$ \\
\hline Tratamento mutilador & $\begin{array}{l}\text { Prevalência obtida através da pergunta "Fez extração na sua unidade } \\
\text { de saúde?" }\end{array}$ \\
\hline Ações preventivas individuais & $\begin{array}{l}\text { Prevalência obtida através da pergunta "Fez prevenção (limpeza, aplicação } \\
\text { de flúor) na sua unidade de saúde?" }\end{array}$ \\
\hline Ações preventivas coletivas & $\begin{array}{l}\text { Prevalência de indivíduos obtida através da pergunta "Participou de palestras } \\
\text { sobre saúde bucal?" }\end{array}$ \\
\hline
\end{tabular}

ESB: equipe de saúde bucal; ESF: Estratégia Saúde da Família.

06.033/2006, apresentando-se de acordo com a Resolução 196/96 do Conselho Nacional de Saúde.

\section{Resultados}

A população prevista para compor a amostra era de 75.148 indivíduos. Foram percorridos 16.457 domicílios, gerando um banco de dados de 59.221 indivíduos $(78,8 \%$ da amostra prevista). Dos 120 setores censitários previstos para compor cada grupo, de acordo com a descrição da amostra na Figura 1, alguns setores de áreas cobertas pelo modelo ESF com saúde bucal e, consequentemente, o mesmo número de setores controle deixaram de ser investigados devido a problemas operacionais. A análise, portanto, refere-se aos dados coletados em 214 setores, sendo 107 em cada grupo. Do total de indivíduos entrevistados, 30.165 pertenciam às áreas com cobertura do ESF com saúde bucal (Modelo 1). O restante pertencia às áreas com modelo tradicional ou sem cobertura de saúde bucal (Modelo 2). A perda final de indivíduos $(21,2 \%)$ está próxima do limite permitido para o desenho do estudo proposto, o qual previu uma taxa de não resposta de $20 \%$ (Figura 1).

Também foi assegurada uma distribuição equilibrada da amostra entre as áreas do Modelo 1 e do Modelo 2, mantendo o que fora previsto com o emparelhamento intencional dos setores. 


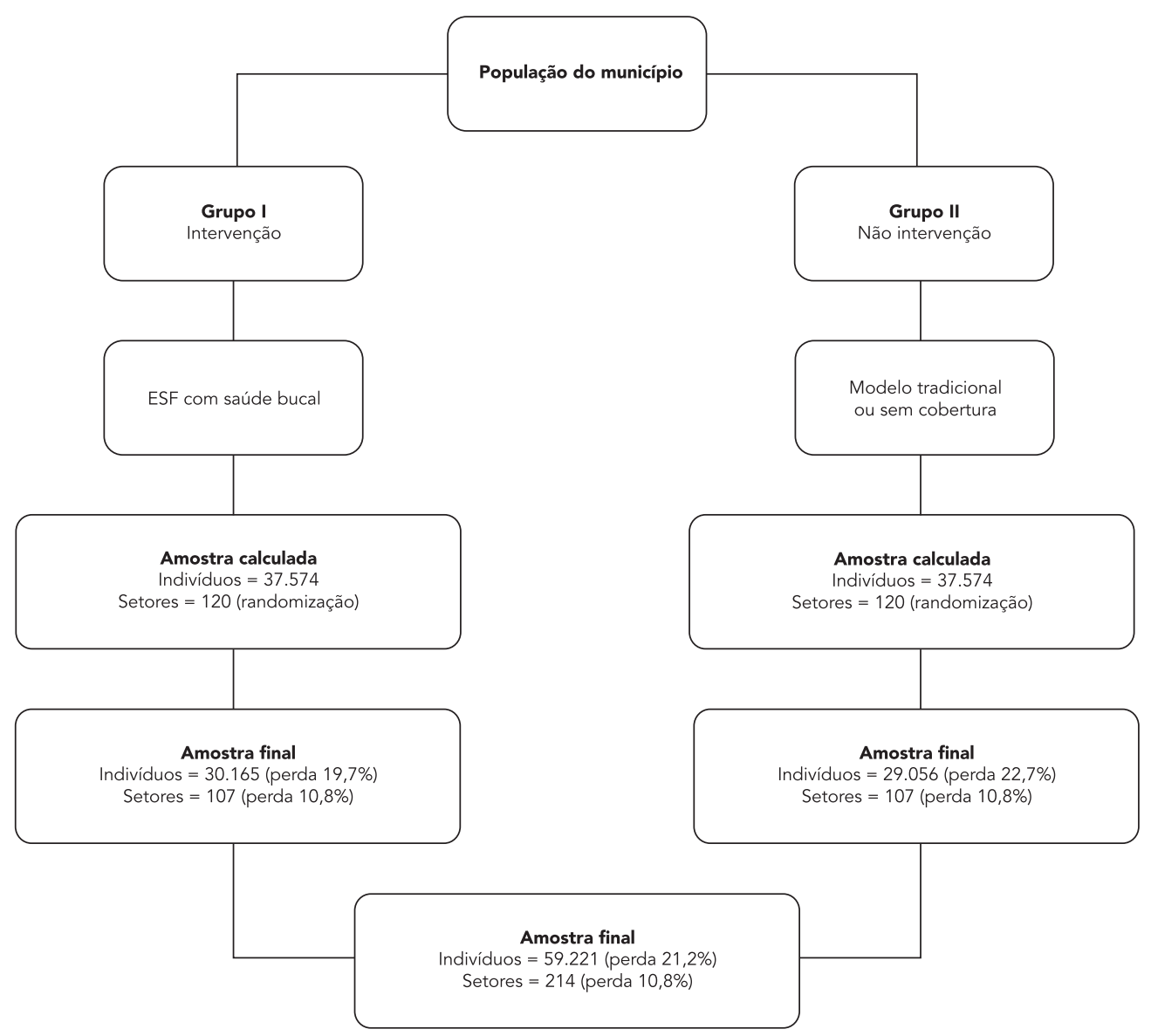

ESF: Estratégia Saúde da Família.

\section{Distribuição da amostra segundo sexo e idade}

Da população pesquisada em áreas com cobertura ESF com SB, 46,7\% eram do sexo masculino, e $53,5 \%$, do sexo feminino. Na área não coberta, $46,4 \%$ eram do sexo masculino, e $53,6 \%$, do sexo feminino, indicando que não existe diferença entre as áreas com relação ao sexo. O mesmo comportamento é observado quando se compara a faixa etária da amostra em relação aos dois grupos estudados.

\section{Análise do impacto sobre indicadores} de saúde bucal

A Tabela 2 ilustra as frequências de cada indicador de acordo com os modelos estudados, os va- lores de p, as RP e respectivos IC95\%, ajustados para fatores sociodemográficos por intermédio da regressão de Poisson. Isso significa que, em linhas gerais, as diferenças entre as áreas cobertas pelo Modelo 1 e pelo Modelo 2, com significância estatística, podem ser interpretadas independentemente de um possível efeito de confusão. Os símbolos indicam, tendo como referência o Modelo 1, um impacto positivo $(\uparrow)$, ausência de efeito $(\bullet)$ ou impacto negativo $(\downarrow)$.

Esses resultados expressos na Tabela 2 podem ser lidos a partir de duas perspectivas. A primeira delas é observar os dados horizontalmente, em que um dado município pode ser analisado isoladamente e comparado aos demais com relação ao seu desempenho frente aos indicadores. Um segundo modo de interpretar os dados é observálos verticalmente, tentando entender como um 
Resultados dos indicadores de saúde bucal em áreas com Modelo 1 (SB-PSF) e Modelo 2 nos 12 municípios investigados. Brasil, 2010.

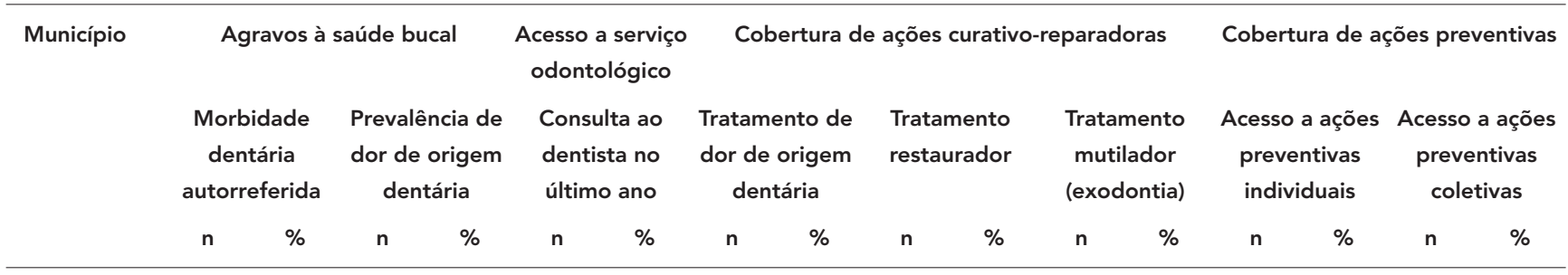

A

\begin{tabular}{|c|c|c|c|c|c|c|c|c|c|c|c|c|c|c|c|c|}
\hline Modelo 1 & 1.460 & 56,8 & 598 & 23,2 & 553 & 21,4 & 168 & 28,1 & 319 & 55,9 & 259 & 45,2 & 267 & 46,4 & 249 & 9,6 \\
\hline Modelo 2 & 1.604 & 64,4 & 558 & 22,4 & 679 & 27,1 & 177 & 31,8 & 408 & 60,1 & 405 & 58,8 & 377 & 55,1 & 313 & 12,7 \\
\hline Valor de $p$ & $<0,001$ & $\uparrow$ & 0,524 & - & $<0,001$ & $\downarrow$ & 0,172 & - & 0,132 & - & $<0,001$ & $\downarrow$ & $<0,001$ & $\downarrow$ & $<0,001$ & \\
\hline
\end{tabular}

$\operatorname{RP}($ IC95\%) $1,15(1,10-1,20) \quad 0,97(0,88-1,08) \quad 1,33(1,19-1,47) \quad 1,14(0,95-1,35) \quad 1,08(0,98-1,19) \quad 1,25(1,11-1,43) \quad 1,25(1,12-1,39) \quad 1,35(1,15-1,61)$ B

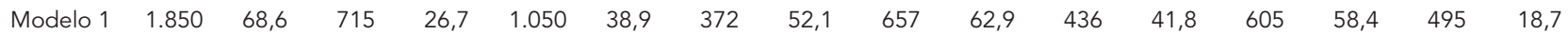

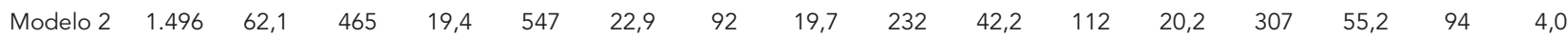

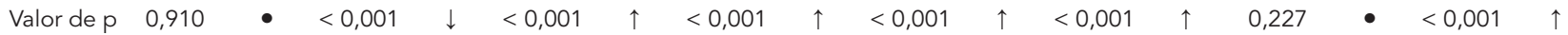

$\operatorname{RP}(\mathrm{IC} 95 \%) \quad 1,00(0,96-1,04) \quad 0,80(0,71-0,89) \quad 0,59(0,54-0,65) \quad 0,38(0,31-0,46) \quad 0,67(0,59-0,76) \quad 0,55(0,44-0,67) \quad 0,94(0,94-1,04) \quad 0,21(0,17-0,26)$ C

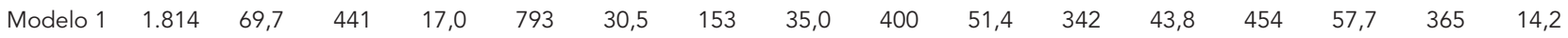

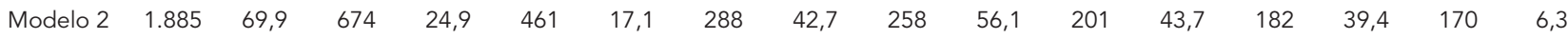

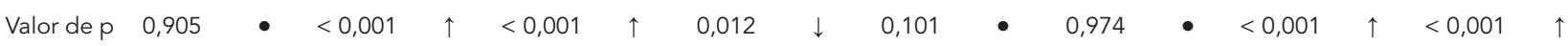
$\operatorname{RP}(\mathrm{IC} 95 \%) \quad 1,00(0,97-1,04) \quad 1,67(1,49-1,89) \quad 0,56(0,51-0,63) \quad 1,22(1,04-1,43) \quad 1,09(0,62-1,22) \quad 1,00(0,88-1,14) \quad 0,70(0,62-0,79) \quad 0,58(0,48-0,71)$ D

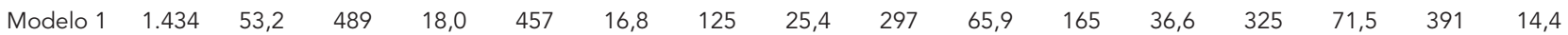

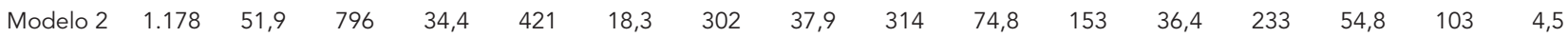

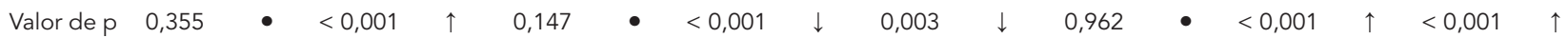
$\operatorname{RP}(\mathrm{IC} 95 \%) \quad 0,98(0,93-1,03) \quad 2,04(1,82-2,22) \quad 1,10(0,97-1,10) \quad 1,49(1,25-1,79) \quad 1,14(1,04-1,25) \quad 1,00(0,83-1,19) \quad 0,78(0,70-0,86) \quad 0,34(0,27-0,43)$ E

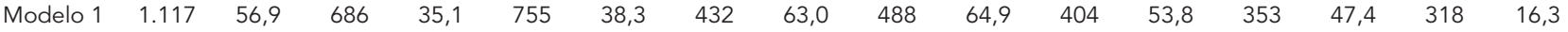

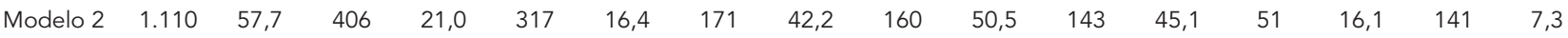

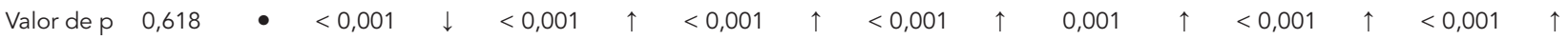
$\operatorname{RP}($ IC95\%) $\quad 1,01(0,96-1,08) \quad 0,66(0,59-0,74) \quad 0,49(0,43-0,54) \quad 0,68(0,60-0,78) \quad 0,80(0,71-0,90) \quad 0,79(0,69-0,91) \quad 0,34(0,26-0,44) \quad 0,38(0,31-0,47)$ $\mathrm{F}$

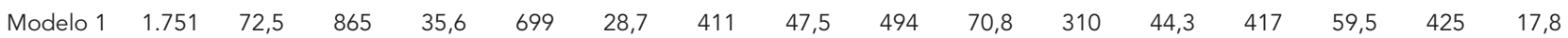

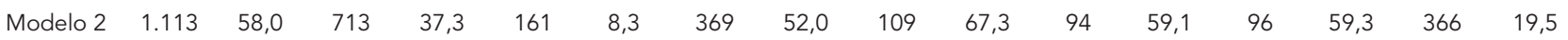

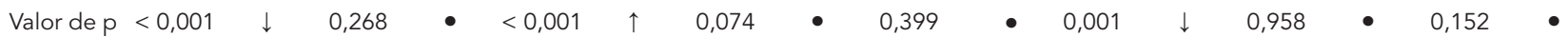
$\operatorname{RP}(\operatorname{IC95\% )} 0,84(0,80-0,88) \quad 1,04(0,97-1,14) \quad 0,28(0,24-0,34) \quad 1,10(0,99-1,20) \quad 0,95(0,85-1,08) \quad 1,35(1,16-1,59) \quad 1,00(0,87-1,15) \quad 1,10(0,97-1,25)$ G

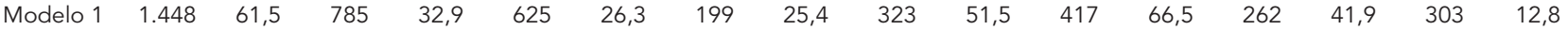

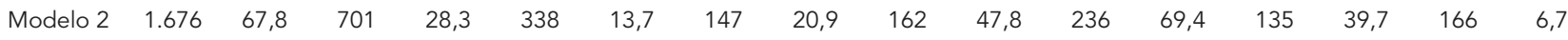

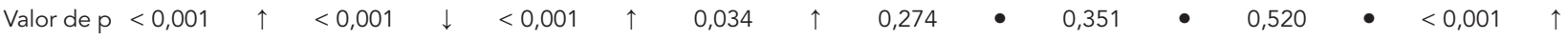
$\operatorname{RP}($ IC95\%) $1,09(1,04-1,14) \quad 0,85(0,78-0,93) \quad 0,52(0,46-0,59) \quad 0,82(0,68-0,99) \quad 0,93(0,81-1,06) \quad 1,04(0,95-1,15) \quad 0,95(0,81-1,12) \quad 0,53(0,45-0,64)$ $\mathrm{H}$

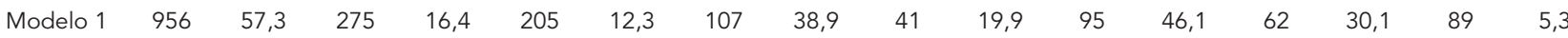

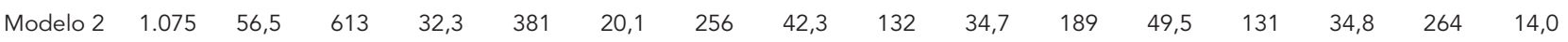

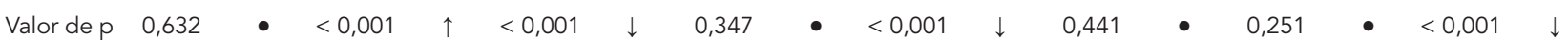
$\operatorname{RP}(\mathrm{IC} 95 \%) \quad 0,99(0,93-1,04) \quad 2,04(1,75-2,38) \quad 1,64(1,41-1,92) \quad 1,09(0,92-1,30) \quad 1,75(1,28-2,38) \quad 1,08(0,87-1,28) \quad 1,16(0,91-1,54) \quad 2,70(2,13-3,33)$ 


\begin{tabular}{|c|c|c|c|c|c|c|c|c|c|c|c|c|c|c|c|c|}
\hline \multirow[t]{3}{*}{ Município } & \multicolumn{4}{|c|}{ Agravos à saúde bucal } & \multirow{2}{*}{\multicolumn{2}{|c|}{$\begin{array}{c}\text { Acesso a serviço } \\
\text { odontológico } \\
\text { Consulta ao } \\
\text { dentista no } \\
\text { último ano }\end{array}$}} & \multicolumn{6}{|c|}{ Cobertura de ações curativo-reparadoras } & \multicolumn{4}{|c|}{ Cobertura de ações preventivas } \\
\hline & \multicolumn{2}{|c|}{$\begin{array}{l}\text { Morbidade } \\
\text { dentária } \\
\text { autorreferida }\end{array}$} & \multicolumn{2}{|c|}{$\begin{array}{l}\text { Prevalência de } \\
\text { dor de origem } \\
\text { dentária }\end{array}$} & & & \multicolumn{2}{|c|}{$\begin{array}{l}\text { Tratamento de } \\
\text { dor de origem } \\
\text { dentária }\end{array}$} & \multicolumn{2}{|c|}{$\begin{array}{l}\text { Tratamento } \\
\text { restaurador }\end{array}$} & \multicolumn{2}{|c|}{$\begin{array}{l}\text { Tratamento } \\
\text { mutilador } \\
\text { (exodontia) }\end{array}$} & \multicolumn{2}{|c|}{$\begin{array}{c}\text { Acesso a ações } \\
\text { preventivas } \\
\text { individuais }\end{array}$} & \multicolumn{2}{|c|}{$\begin{array}{c}\text { Acesso a ações } \\
\text { preventivas } \\
\text { coletivas }\end{array}$} \\
\hline & n & $\%$ & $\mathbf{n}$ & $\%$ & n & $\%$ & $\mathbf{n}$ & $\%$ & $\mathbf{n}$ & $\%$ & $\mathbf{n}$ & $\%$ & $\mathbf{n}$ & $\%$ & $\mathbf{n}$ & $\%$ \\
\hline \multicolumn{17}{|l|}{1} \\
\hline Modelo 1 & 2.344 & 74,7 & 762 & 24,3 & 759 & 24,1 & 231 & 30,3 & 247 & 32,8 & 215 & 28,4 & 516 & 68,1 & 784 & 24,9 \\
\hline Modelo 2 & 2.256 & 76,2 & 734 & 24,6 & 90 & 3,0 & 99 & 13,6 & 31 & 34,8 & 37 & 41,6 & 51 & 57,3 & 188 & 6,3 \\
\hline Valor de p & 0,176 & $\bullet$ & 0,747 & $\bullet$ & $<0,001$ & $\uparrow$ & $<0,001$ & $\uparrow$ & 0,694 & $\bullet$ & 0,010 & $\downarrow$ & 0,102 & $\bullet$ & $<0,001$ & $\uparrow$ \\
\hline RP (IC95\%) & \multicolumn{2}{|c|}{$1,02(0,99-1,05)$} & \multicolumn{2}{|c|}{$1,01(0,93-1,11)$} & \multicolumn{2}{|c|}{$0,13(0,10-0,16)$} & \multicolumn{2}{|c|}{$0,54(0,45-0,65)$} & \multicolumn{2}{|c|}{$1,19(0,79-1,45)$} & \multicolumn{2}{|c|}{$1,43(1,09-1,85)$} & \multicolumn{2}{|c|}{$0,85(0,71-1,03)$} & \multicolumn{2}{|c|}{$0,23(0,20-0,27)$} \\
\hline \multicolumn{17}{|l|}{$J$} \\
\hline Modelo 1 & 1.312 & 51,8 & 744 & 29,3 & 442 & 17,4 & 189 & 25,6 & 284 & 63,3 & 162 & 36,0 & 204 & 45,4 & 277 & 10,9 \\
\hline Modelo 2 & 1.153 & 46,8 & 455 & 18,2 & 417 & 16,7 & 180 & 39,6 & 340 & 81,5 & 238 & 57,2 & 285 & 68,3 & 363 & 14,5 \\
\hline Valor de $\mathrm{p}$ & 0,013 & $\downarrow$ & $<0,001$ & $\downarrow$ & 0,484 & $\bullet$ & $<0,001$ & $\downarrow$ & $<0,001$ & $\downarrow$ & $<0,001$ & $\downarrow$ & $<0,001$ & $\downarrow$ & $<0,001$ & $\downarrow$ \\
\hline RP (IC95\%) & \multicolumn{2}{|c|}{$0,93(0,88-0,99)$} & \multicolumn{2}{|c|}{$0,65(0,58-0,74)$} & \multicolumn{2}{|c|}{$0,96(0,85-1,09)$} & \multicolumn{2}{|c|}{$1,54(1,32-1,85)$} & \multicolumn{2}{|c|}{$1,28(1,19-1,41)$} & \multicolumn{2}{|c|}{$1,61(1,41-1,89)$} & \multicolumn{2}{|c|}{$1,56(1,39-1,75)$} & $1,47(1,2$ & $8-1,69)$ \\
\hline K & & & & & & & & & & & & & & & & \\
\hline Modelo 1 & 1.634 & 67,5 & 586 & 24,0 & 654 & 26,7 & 189 & 33,6 & 299 & 45,8 & 255 & 39,1 & 294 & 44,8 & 317 & 13,0 \\
\hline Modelo 2 & 1.717 & 72,6 & 765 & 32,3 & 135 & 5,7 & 127 & 17,9 & 83 & 61,5 & 88 & 65,2 & 49 & 36,3 & 64 & 2,7 \\
\hline Valor de $p$ & 0,001 & $\uparrow$ & $<0,001$ & $\uparrow$ & $<0,001$ & $\uparrow$ & $<0,001$ & $\uparrow$ & $<0,001$ & $\downarrow$ & $<0,001$ & $\downarrow$ & 0,084 & $\bullet$ & $<0,001$ & $\uparrow$ \\
\hline RP (IC95\%) & $1,06(1, \mathrm{C}$ & $3-1,11)$ & $1,37(1,2$ & $5-1,49)$ & $0,21(0$, & $7-0,25)$ & $0,49(0,4$ & $0-0,60)$ & $1,33(1,1$ & $4-1,56)$ & $1,59(1,3$ & $5-1,85)$ & $0,81(0,6$ & $4-1,03)$ & $0,19(0,1$ & $5-0,25)$ \\
\hline $\mathrm{L}$ & & & & & & & & & & & & & & & & \\
\hline Modelo 1 & 1.221 & 59,7 & 483 & 23,9 & 469 & 23,9 & 156 & 37,2 & 275 & 61,8 & 181 & 44,0 & 117 & 28,4 & 191 & 9,5 \\
\hline Modelo 2 & 1.325 & 62,2 & 676 & 31,9 & 93 & 4,3 & 62 & 9,2 & 53 & 57,0 & 43 & 46,2 & 50 & 53,8 & 220 & 10,3 \\
\hline Valor de p & 0,098 & $\bullet$ & $<0,001$ & $\uparrow$ & $<0,001$ & $\uparrow$ & $<0,001$ & $\uparrow$ & 0,406 & $\bullet$ & 0,697 & $\bullet$ & $<0,001$ & $\downarrow$ & 0,396 & $\bullet$ \\
\hline RP (IC95\%) & $1,04(0$, & $9-1,10)$ & $1,33(1,2$ & $2-1,54)$ & $0,13(0$, & $0-0,17)$ & $0,23(0,1$ & $7-0,31)$ & $0,93(0,7$ & $6-1,11)$ & $1,05(0,8$ & $3-1,33)$ & $2,04(1,6$ & $1-2,560$ & $1,09(0,9$ & $0-1,32)$ \\
\hline
\end{tabular}

Nota: Para cada análise, valores de p e razão de prevalência (RP) ajustada pela regressão de Poisson e respectivos intervalos de confiança (IC95\%). Ajustes feitos a partir da regressão de Poisson, com as variáveis "idade", "sexo", "renda per capita", "número de bens" e "escolaridade da mãe". Dependendo do desfecho, uma ou mais dessas variáveis fizeram parte do modelo final. Os símbolos indicam impacto positivo ( $\uparrow$ ), ausência de impacto (•) e impacto negativo $(\downarrow)$, tendo como referência o Modelo 1.

determinado indicador se comporta, em termos de impacto, nos 12 municípios pesquisados. As Figuras 2 e 3 tentam sintetizar essas duas leituras. A Figura 2 sintetiza os resultados tendo, como referência, o número de indicadores em cada categoria para cada um dos 12 municípios avaliados, e a Figura 3 ilustra a distribuição dos municípios em relação a cada um dos 8 indicadores.

Pode-se observar que, em nenhum município, houve um impacto global da ESB-ESF (Modelo 1), refletindo-se em um impacto positivo em todos os 8 indicadores avaliados. O município "E" apresentou os melhores resultados, com impacto positivo em 6 indicadores, porém, no outro extremo, o município "J" apresentou impacto negativo em 7 dos 8 indicadores. Desse modo, não há como estabelecer um padrão uniforme nos municípios avaliados com relação à avaliação de impacto.
Tomando como base a análise para cada indicador especificamente, pode-se observar, pela análise da Figura 3, que os indicadores relativos a acesso e cobertura ("acesso a serviço odontológico”, "acesso a ações preventivas coletivas” e "tratamento da dor de dente") foram os que apresentaram maior número de municípios com resultados positivos.

A diferença encontrada no indicador "acesso ao dentista” entre as áreas foi significativa e favorável ao Modelo 1 em oito municípios. Nos quatro restantes, metade apresentou impacto negativo, e metade, ausência de impacto.

$\mathrm{O}$ acesso a ações preventivas coletivas teve um comportamento semelhante, diferindo em um município a mais com impacto negativo. $\mathrm{O}$ "tratamento da dor de dente" apresentou metade dos municípios com impacto positivo, três com impacto negativo e três com ausência de impacto. 
Figura 2

Distribuição dos 8 indicadores com relação ao seu resultado (positivo, ausência ou negativo) nos 12 municípios estudados. A ordem foi estabelecida da esquerda para a direita, considerando o melhor até o pior resultado.

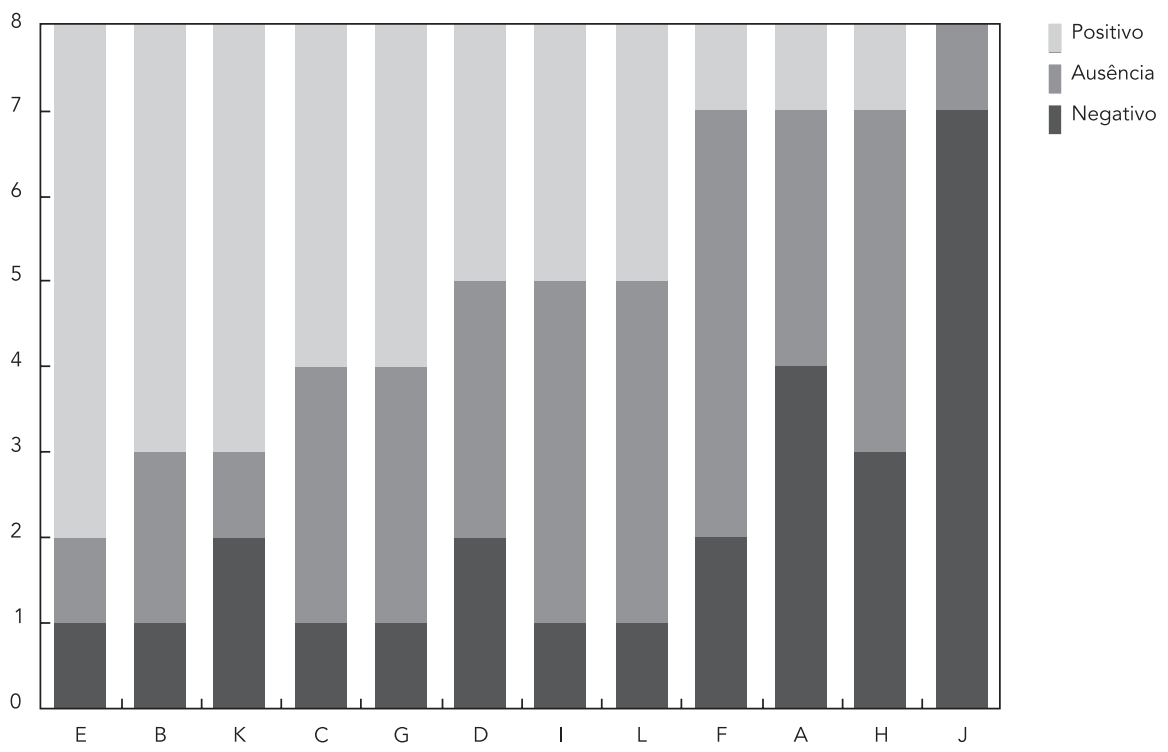

Figura 3

Distribuição dos 12 municípios analisados com relação aos resultados encontrados para os indicadores de saúde bucal utilizados. A ordem foi estabelecida de baixo para cima, considerando o pior até o melhor resultado.

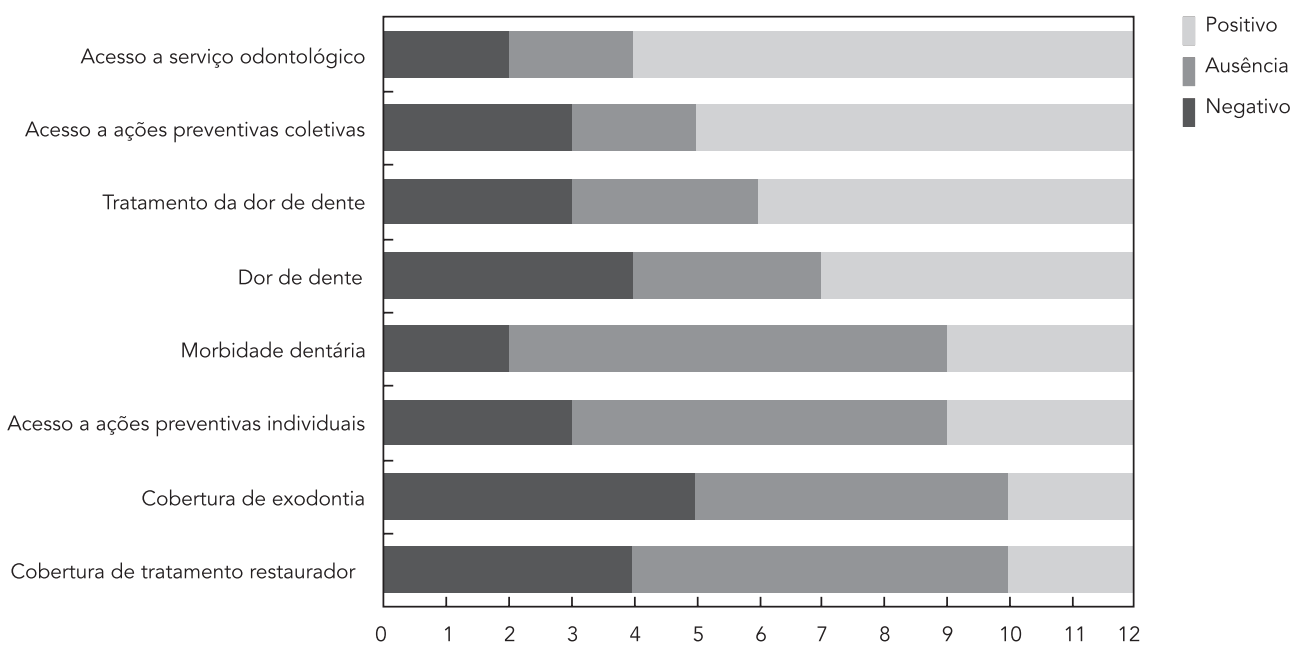


No caso dos indicadores relativos aos agravos ("dor de dente" e "morbidade dentária autorreferida”), os resultados tenderam mais para ausência de impacto ou impacto negativo.

Com relação à "dor de origem dentária”, foram observados resultados positivos em cinco municípios. Em três deles, não existiu diferença significativa entre as áreas; e em quatro, não foi possível apontar uma tendência de redução da prevalência desse agravo em áreas do Modelo 1.

Já com relação à "morbidade dentária autorreferida", verificou-se um impacto positivo nas áreas cobertas pela ESB-ESF nos municípios "A", "G" e "K". Os municípios "F" e "J" apresentaram um resultado inverso, ou seja, as áreas com Modelo 1 apresentaram maior prevalência desse agravo quando comparadas às áreas com Modelo 2. Os demais municípios apresentaram percentuais semelhantes entre as áreas. É possível constatar, também, que, na quantidade de necessidade acumulada, o percentual mínimo encontrado foi de $46 \%$, e, na maioria dos casos, mais da metade dos entrevistados tinham necessidade de algum tipo de intervenção odontológica. Em números absolutos, significa um montante de 35.929 indivíduos, ou seja, $63 \%$ da amostra.

Finalmente, para os indicadores relativos à cobertura de ações preventivas individuais, de exodontia e de tratamento restaurador, a tendência maior foi de ausência de impacto (6, 5 e 6 municípios, respectivamente) ou mesmo impacto negativo (3, 5 e 4 municípios, respectivamente).

De uma maneira geral, os resultados indicam uma situação preocupante em, pelo menos, sete municípios (D, I, L, F, A, H e J) onde se observa predominância de uma ausência de impacto ou impacto negativo nos indicadores (Tabela 2 e Figura 2).

\section{Discussão}

A análise do impacto dos municípios em relação aos indicadores, sintetizada na Figura 2, pode conduzir à leitura de que, em apenas três municípios, houve uma maioria de efeitos positivos em relação aos negativos ou ausência de efeito (E, B e $\mathrm{K})$. Em dois deles, houve igual número de indicadores com resultado positivo e negativo (C e G); e, nos outros sete, há uma tendência maior para ausência de efeito ou efeito negativo. Apenas pela observação dos números da tabela, pode-se inferir um fraco desempenho da incorporação da saúde bucal na ESF, considerando os resultados gerais nos 12 municípios pesquisados. É possível, no entanto, ponderar que resultados positivos em três municípios, dentre 12 pesquisados, podem ser vistos sob uma lente mais otimista, tendo em conta o tempo de implantação da ESF, as dificuldades na gestão e a conhecida dívida assistencial acumulada durante décadas 14 .

Assim, dada a ausência de parâmetros claros e tendo em conta a subjetividade exposta no parágrafo anterior, os autores deste estudo consideram que esses resultados devem ser interpretados como um fraco desempenho da saúde bucal na ESF nos municípios avaliados e devem ser vistos com preocupação por gestores e formuladores de políticas de saúde. Tal assertiva se baseia nos seguintes pontos: (a) a Estratégia Saúde da Família, com a incorporação das equipes de saúde bucal desde 2001, tem proporcionado um aumento considerável na cobertura dos serviços de saúde bucal, tanto do ponto de vista do aumento no número de profissionais quanto das horas efetivamente trabalhadas 21 ; (b) os profissionais da ESF têm regime de trabalho diferenciado, com diferenças salariais e de condições de trabalho marcantes em comparação com profissionais “não-ESF”; (c) o processo de trabalho preconizado para a saúde bucal na ESF pressupõe uma abordagem que proporcione a ampliação do acesso coletivo às ações de promoção, prevenção e recuperação da saúde bucal, segundo as Diretrizes da Política Nacional de Saúde Bucal 22; (d) os indicadores avaliados neste estudo são todos passíveis de serem afetados por intervenções assistenciais no espaço de tempo considerado (em média, três anos de implantação).

Desse modo, é importante especular sobre quais os fatores que estão fazendo com que equipes de saúde bucal com melhor remuneração (e com mais horas disponíveis), mais presentes nas áreas adscritas e com um modelo de prática que deveria ser pautado na promoção, prevenção e recuperação da saúde bucal praticamente não se diferenciem na tarefa de reduzir o sofrimento bucal.

A análise em sentido vertical da Tabela 2, sintetizada na Figura 3, pode dar uma pista. É possível notar que o maior número de municípios com desempenho positivo ocorre nos três indicadores relativos ao acesso e à cobertura dos serviços. Os dois indicadores de agravos (dor de dente e morbidade referida), por sua vez, têm um menor número de municípios com efeito positivo. Sintomaticamente, o acesso a ações preventivas individuais também tem fraco desempenho. Conforme já apontavam Souza \& Roncalli 15, um aumento no acesso a serviços odontológicos não se traduz, num primeiro momento, em reversão do modelo de atenção à saúde bucal caracterizado por ser pouco resolutivo, mutilador e, portanto, centrado na doença. Nessa mesma linha, concluem Pereira et al. ${ }^{23}$, analisando o impacto 
sobre indicadores de agravos e cobertura de saúde bucal depois de decorridos mais de um ano de implantação da ESB em áreas com e sem cobertura de ESF no Município de Natal. Uma primeira hipótese para a ocorrência desse quadro é que a inclusão das ESB na ESF não se traduziu em uma mudança no processo de trabalho.

Ainda assim, os resultados deste estudo, considerando o efeito sobre o acesso a serviços odontológicos, podem ser considerados favoráveis. Em uma pesquisa de base populacional, conduzida por Rocha \& Góes 24 , em Campina Grande (Paraíba), com uma amostra composta por pessoas acima de 18 anos, não foi encontrada associação, após os ajustes, entre residir em uma área coberta ou não pela ESF com relação ao acesso aos serviços de saúde bucal.

Do ponto de vista da cobertura por ações preventivas individuais, apenas três municípios apresentaram resultados positivos em áreas com cobertura da estratégia. $\mathrm{O}$ acesso a ações preventivas individuais, portanto, aparece entre os piores resultados, sendo que essa situação vai de encontro às recomendações presentes nas Diretrizes da Política Nacional de Saúde Bucal 25 . No entendimento de Almeida \& Ferreira 26 , a regularidade e a frequência do procedimento preventivo em saúde bucal ainda são informações escassas, o que as motivou verificar, não só a incorporação das práticas preventivas e educativas, como também a existência de instrumentos de avaliação dessas atividades. Por meio de entrevista estruturada com 80 dentistas da ESF e análise documental no SIA-SUS (Sistema de Informações Ambulatoriais do SUS) e no SIAB, identificaram que as atividades individuais predominantes são a orientação de higiene bucal e a aplicação tópica de flúor, correspondendo a $87,5 \%$ e $95 \%$, respectivamente. Nos registros do SIA-SUS, as atividades preventivas representaram $41 \%$ do total de procedimentos. Mais de $70 \%$ dos dentistas relataram não ter instrumento de avaliação ou não ter como avaliar o impacto das ações. Nesse mesmo estudo concluem que, apesar de a ESF se apresentar como um modelo reestruturador, verifica-se que ainda existe reprodução de métodos tradicionais.

Se compararmos os resultados encontrados nas atividades individuais com o bom resultado do acesso às ações preventivas coletivas (sete municípios apresentaram resultados positivos em áreas cobertas pela ESF), à primeira vista, pode parecer uma priorização das ações preventivas coletivas em detrimento das ações individuais em áreas cobertas pela ESF, porém os percentuais estão bem abaixo (máximo de 20\%) dos obtidos nas atividades individuais (acima de $30 \%)$.
Finalmente, há que se considerar, também, a base de organização dos municípios, a qualidade das informações básicas tais como percentual de cobertura das ESF com e sem ESB, ausência, em muitos casos, de uma base cartográfica atualizada com os dados de saúde bucal e lentidão dos processos, o que inviabilizou a operacionalização e ou término da coleta nos demais municípios originalmente previstos no projeto. A execução de um estudo seccional pressupõe trabalhar com as limitações de toda pesquisa de campo, tais como o momento político, alta rotatividade de gestores, associada à limitação do tempo em executar os projetos, o que interferiu no recorte da amostra.

\section{Considerações finais}

Sem dúvida, a ESF se constitui em uma estratégia ímpar na reorganização da atenção básica no Brasil desde sua implantação oficial a partir de 1994. O presente trabalho, feito em 12 municípios com mais de 100 mil habitantes do Nordeste, não demonstra a ausência de efeito da ESBESF per se, conforme também foi demonstrado anteriormente por Pereira et al. ${ }^{23}$.

Este estudo não teve como proposta investigar os modelos assistenciais e os processos de trabalho em cada município, mas comparar indicadores em áreas submetidas ou não a uma política pública específica, nesse caso, a inclusão de equipes de saúde bucal na ESF. Desse modo, as eventuais causas relacionadas aos processos de implantação devem ser objeto de investigações futuras. Contudo, os dados encontrados demonstram que a incorporação dessa ação pública está produzindo resultados que poderiam ser melhores, tendo em conta o investimento e a proposta de inversão de modelo. E há claros indícios de que o modo como as estratégias são estabelecidas em cada local seja fator determinante para a ocorrência ou não de impacto sobre os indicadores.

Ademais, é oportuno destacar os bons resultados da ESF na Atenção Primária em Saúde sobre indicadores tradicionais como a mortalidade infantil, a desnutrição e as internações por causas preveníveis, conforme foram demonstrados pelos estudos de Macinko et al. 4, Aquino et al. 5, Monteiro et al. 6, Rocha \& Soares 7, Veloso \& Araújo 8 e Goldbaum et al. ${ }^{9}$. Há, portanto, alguma característica peculiar do modelo de atenção em saúde bucal que faz com que não sejam obtidos impactos semelhantes.

É pertinente, também, alertar para as limitações deste trabalho. Como critério para inclusão de áreas cobertas, foram consideradas apenas as 
áreas onde efetivamente se deu a implantação das ESB pelo período mínimo de um ano (ao fim do estudo, o tempo médio de cobertura encontrado para as áreas cobertas pela ESB foi de três anos). Trata-se de um corte arbitrário e poderia ser considerado pouco tempo para se realizar uma avaliação de impacto, entretanto, conforme discutimos anteriormente, ainda não temos esse parâmetro. Além disso, mesmo considerando que a avaliação a partir de indicadores autorreferidos já está consolidada na literatura 27 , outros estudos que incluam indicadores de morbidade a partir de uma avaliação por parte do profissional devem ser conduzidos. E, por fim, o próprio desenho do estudo, pelo fato de se caracterizar como observacional e transversal, também apresenta limitações inerentes ao emprego desse tipo de delineamento na avaliação de programas.

Do ponto de vista da articulação desses resultados com a atual política de saúde, deve-se destacar que a Política Nacional de Saúde Bucal 25 é clara ao afirmar, em suas diretrizes, que as ações e serviços devem resultar de um adequado conhecimento da realidade de saúde de cada localidade para, a partir disso, construir uma prática efetivamente resolutiva. Segue frisando que é imprescindível, em cada território, aproximar-se das pessoas e tentar conhecê-las, estudar suas condições de vida, as representações e as concepções que têm acerca de sua saúde, seus hábitos e as providências que tomam para resolver seus problemas quando adoecem, bem como o que fazem para evitar enfermidades. Nesse sentido, entendemos que os indicadores utilizados são limitados para avaliar a qualidade dos processos de implantação, e, para tanto, existem outras formas de pesquisas que buscam explicar os êxitos e fracassos de um programa.

Ressalte-se, ainda, que as mudanças em um modelo assistencial, no caso o odontológico, segundo Souza \& Roncalli 15, dependem, dentre outros fatores, do contexto político, da organização dos serviços, de processos de capacitação e do envolvimento da equipe.

Desse modo, a Academia (entendida aqui como o conjunto de instituições voltadas para o ensino e para a pesquisa) tem tido papel importante nos últimos anos, realizando estudos que procuram promover e reafirmar o SUS como a política de saúde de todos os brasileiros. Embora reconhecendo que a avaliação é um atributo que se estabelece primordialmente na esfera dos municípios, a função da universidade como geradora de conhecimento se estrutura em um caminho duplo: por um lado, gera informações importantes para as ações de planejamento e avaliação e, por outro, pode (e deve) proporcionar empoderamento dos serviços no sentido de incorporar tais práticas às suas rotinas e protocolos de gestão.

\section{Resumo}

Este estudo objetivou verificar o impacto da Estratégia Saúde da Família (ESF) sobre a saúde bucal na população do Nordeste do Brasil de 12 municípios com mais de 100 mil habitantes. Tomou-se, como referência, 20 setores censitários, 10 inseridos em áreas cobertas pela saúde bucal na ESF há, pelo menos, um ano, emparelhados com 10 setores de áreas não cobertas. A amostra foi de 59.221 indivíduos. Foram considerados três efeitos possíveis: negativo, positivo e ausência de efeito sobre os desfechos, com base na razão de prevalência ajustada pela regressão de Poisson. Com relação aos resultados encontrados, verificou-se que, tanto o "aces- so a ações preventivas coletivas" quanto o "tratamento da dor de dente" apontam para um efeito positivo no modelo de prática da saúde bucal na ESF Porém, os desfechos "cobertura de exodontia" e "tratamento restaurador" demonstraram efeito negativo ou sem efeito na maioria dos municípios pesquisados. Há indícios de que os resultados obtidos refletem os modelos de organização locais da saúde bucal na ESF.

Programa Saúde da Família; Saúde Bucal; Avaliação de Serviço de Saúde 


\section{Colaboradores}

C. R. S. Pereira participou das etapas de coleta de dados, análise dos dados, construção e redação final do texto. A. G. Roncalli participou da elaboração e coordenação do projeto, do delineamento amostral e da análise dos dados, construção e redação final do texto. M. C. T. Cangussu conduziu a pesquisa em dois municípios, participou da análise dos dados, construção e redação final do texto. L. R. A. Noro conduziu a pesquisa em dois municípios, participou da análise dos dados, construção e redação final do texto. A. A. R. Patrício conduziu a pesquisa em três municípios, participou da análise dos dados, construção e redação final do texto. K. C. Lima participou da elaboração e coordenação do projeto, do delineamento amostral e da análise dos dados, construção e redação final do texto.

Programa Saúde da Família; Saúde Bucal; Avaliação de Serviços de Saúde

\section{Referências}

1. Travassos C, Novaes HMD. Investigação em serviços de saúde. Cad Saúde Pública 2004; 20 Suppl 2:S144-5.

2. Brasil. Lei $\mathrm{n}^{\circ}$. 8.080, de 19 de setembro de 1990 . Dispõe sobre as condições para a promoção, proteção e recuperação da saúde, a organização e o funcionamento dos serviços correspondentes e dá outras providências. Diário Oficial da União 1990; 20 set.

3. Ministério da Saúde. Portaria no ${ }^{\circ}$ 1.444/GM. Cria o incentivo de Saúde Bucal para o financiamento de ações e da inserção de profissionais de Saúde Bucal no Programa de Saúde da Família. Diário Oficial da União 2000; 29 dez.

4. Macinko J, Souza MFM, Guanais FC, Simões CCS. Going to scale with community-based primary care: an analysis of the family health program and infant mortality in Brazil, 1999-2004. Soc Sci Med 2007; 65:2070-80.

5. Aquino R, Oliveira NF, Barreto ML. Impact of the family health program on infant mortality in Brazilian municipalities. Am J Public Health 2009; 99:87-93.

6. Monteiro CA, Benicio MHD, Konno SC, Silva ECF, Lima ALL, Conde WL. Causas do declínio da desnutrição infantil no Brasil, 1996-2007. Rev Saúde Pública 2009; 43:35-43.

\section{Agradecimentos}

Os autores agradecem às instituições financiadoras (CNPq pelo fomento e CAPES pela bolsa), às Secretarias Municipais de Saúde dos municípios, pelo apoio à realização da pesquisa e às universidades que participaram da execução do estudo.
7. Rocha R, Soares RR. Evaluating the impact of community-based health interventions: evidence from Brazil's Family Health Program. Bonn: Institute for the Study of Labor; 2009. (Discussion Paper Series).

8. Veloso RC, Araújo MRN. Avaliação da resolutividade do programa saúde da família em municípios de pequeno porte no Estado de Minas Gerais. Rev APS 2009; 12:238-43.

9. Goldbaum M, Gianini RJ, Novaes HMD, César CLG. Utilização de serviços de saúde em áreas cobertas pelo Programa Saúde da Família (Qualis) no Município de São Paulo. Rev Saúde Pública 2005; 39:90-9.

10. Baldani MH, Fadel CB, Possamai T, Queiroz MGS. A inclusão da odontologia no Programa Saúde da Família no Estado do Paraná, Brasil. Cad Saúde Pública 2005; 21:1026-35.

11. Andrade KLC, Ferreira EF. Avaliação da inserção da odontologia no Programa Saúde da Família de Pompeu (MG): a satisfação do usuário. Ciênc Saúde Coletiva 2006; 11 Suppl 1:123-30.

12. Oliveira JLC, Saliba NA. Atenção odontológica no Programa de Saúde da Família de Campos dos Goytacazes. Ciênc Saúde Coletiva 2005; 10 Suppl: 297-302. 
13. Santos AM, Assis MMA. Da fragmentação à integralidade: construindo e (des) construindo a prática de saúde bucal no Programa de Saúde da Família (PSF) de Alagoinhas, BA. Ciênc Saúde Coletiva 2006; 11:53-61.

14. Martelli PJL, Cabral APS, Pimentel FCM, Maceo CLSV, Monteiro ISS, Silva SF. Análise do modelo de atenção à saúde bucal em municípios do Estado de Pernambuco. Ciênc Saúde Coletiva 2008; 13:1669-74.

15. Souza TMS, Roncalli AG. Saúde bucal no Programa Saúde da Família: uma avaliação do modelo assistencial. Cad Saúde Pública 2007; 23:2727-39.

16. Almeida Filho N, Rouquayrol Z. Desenhos de pesquisa epidemiológica. In: Rouquayrol Z, Almeida Filho N, organizadores. Epidemiologia \& saúde. Rio de Janeiro: Medsi; 1999. p. 149-70.

17. Instituto Brasileiro de Geografia e Estatística. Base de dados por setor censitário [CD-ROM]. Rio de Janeiro: Instituto Brasileiro de Geografia e Estatística; 2005.

18. Altman DG. Practical statistics for medical research. New York: Chapman \& Hall/CRC; 1990.

19. Roncalli AG, Lima KC. Impacto do Programa Saúde da Família sobre indicadores de saúde da criança em municípios de grande porte da região Nordeste do Brasil. Ciênc Saúde Coletiva 2006; 10:297-302.

20. Barros FC, Victora CG. Epidemiologia da saúde infantil: um manual para diagnósticos comunitários. São Paulo: Editora Hucitec/Fundo das Nações Unidas para a Infância; 1998.

21. Costa JFR, Chagas LD, Silvestre RM, organizadores. A política nacional de saúde bucal do Brasil: registro de uma conquista histórica. Brasília: Organização Pan-Americana da Saúde; 2006. (Série Técnica: Desenvolvimento de Sistemas e Serviços de Saúde, 11).
22. Ministério da Saúde. Portaria nº. 267, de 7 de março. Aprova as Normas e Diretrizes de Inclusão da Saúde Bucal na Estratégia do Programa de Saúde da Família (PSF). Diário Oficial da União 2001; 7 mar.

23. Pereira CRS, Patrício AAR, Araújo FAC, Lucena EES, Lima KC, Roncalli AG. Impacto da estratégia saúde da família com equipe de saúde bucal sobre a utilização de serviços odontológicos. Cad Saúde Pública 2009; 25:985-96.

24. Rocha RACP, Góes PSA. Comparação do acesso aos serviços de saúde bucal em áreas cobertas e não cobertas pela Estratégia Saúde da Família em Campina Grande, Paraíba, Brasil. Cad Saúde Pública 2008; 24:2871-80.

25. Coordenação Nacional de Saúde Bucal, Departamento de Atenção Básica, Secretaria de Atenção à Saúde, Ministério da Saúde. Diretrizes da Política Nacional de Saúde Bucal. http://bvsms.saude.gov. br/bvs/publicacoes/politica_nacional_brasil_sor ridente.pdf (acessado em 30/Jun/2010).

26. Almeida GCM, Ferreira MAF. Saúde bucal no contexto do Programa Saúde da Família: práticas de prevenção orientadas ao indivíduo e ao coletivo. Cad Saúde Pública 2008; 24:2131-40.

27. Cesar CLG, Figueiredo GM, Westphal MF, Cardoso MRA, Costa MZA, Gattás VL. Morbidade referida e utilização de serviços de saúde em localidades urbanas brasileiras: metodologia. Rev Saúde Pública 1996; 30:153-60.

Recebido em 08/Fev/2011

Versão final reapresentada em 20/Out/2011 Aprovado em 08/Nov/2011 